\title{
Das Deutsch der Migranten
}

\section{Bericht von der 48. Jahrestagung des Instituts für Deutsche Sprache}

\author{
von Nikolina Pustički
}

Unter dem Titel „Das Deutsch der Migranten“ stand vom 13. bis 15. März 2012 die 48. IDS-Jahrestagung, welche im Musensaal des Congress Center Rosengarten der Stadt Mannheim stattfand. Sie umfasste knapp 500 Teilnehmer aus 27 Ländern. Nach der Eröffnung durch Ludwig M. Eichinger, Direktor des Instituts für Deutsche Sprache in Mannheim, und der BegrüBung der Mannheimer Bürgermeisterin für Bildung, Jugend, Sport und Gesundheit, Ulrike Freundlieb, folgte die Einführung in das Thema der Tagung durch den Hauptorganisator und Leiter der Abteilung Pragmatik, Arnulf Deppermann.

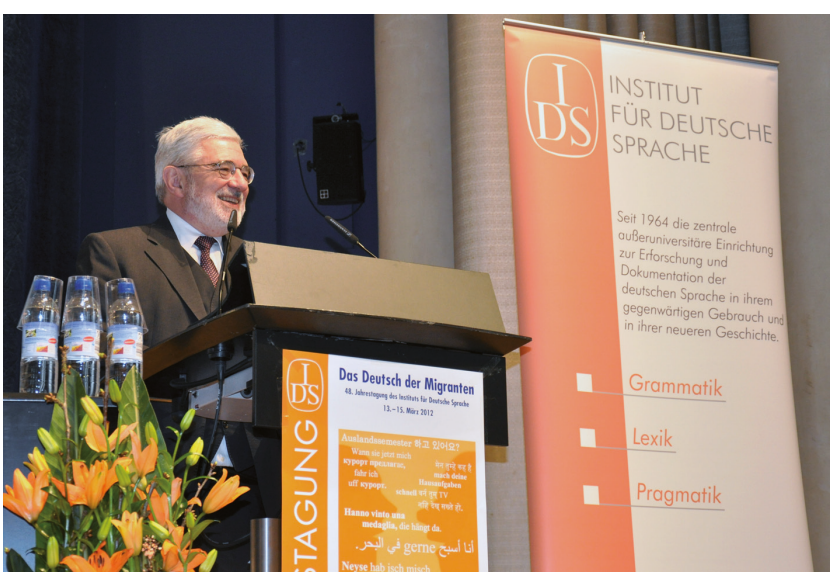

Eröffnungsrede des Direktors des IDS, Prof. Dr. Dr. h.c. mult. Ludwig M. Eichinger

Zum ersten thematischen Schwerpunkt der Jahrestagung „Ethnolekte und Mehrsprachigkeit“ referierte Peter Auer (Freiburg) unter dem Titel „Ethnische Marker im Deutschen zwischen Varietät und Stil“". Auer präsentierte anhand neu erhobener Daten erste Ergebnisse einer Untersuchung zu Sprechweisen türkischstämmiger Jugendlicher in Stuttgart und verwies darauf, dass viele Merkmale, die u. a. bereits in anderen Daten zum primären Ethnolekt der 1990er Jahre zu finden sind, auch im neuen sprachlichen Material nachweisbar sind. Auer verwies aber auch auf neue Erkenntnisse zu Veränderungen im Bereich des Konsonantismus, die im autochthonen Deutschen nicht üblich sind. Auer betonte letztlich, dass die untersuchten Merkmale als auch die mit Selbstbezeichnung wie ,Kiezdeutsch“ oder ,Ghettosprache' benannten ,Sprachen` Gruppen von sozialen Stilen sind, die von
Jugendlichen geformt werden und primär mit einer gesellschaftlichen Selbst- und Fremdpositionierung einhergehen.

Unter dem Titel „Contemporary urban vernaculars“ widmete sich Ben Rampton (London) in seinem Vortrag multiethnisch geprägten Gesprächsstilen und Language Crossing von Sprechern im mittleren Erwachsenenalter mit Migrationshintergrund in West London. Auf der Grundlage empirischer Sprachdaten erläuterte Rampton, dass die Probanden auch über ihr Jugendalter hinweg ihre multi-ethnischen Repertoires beibehielten. Daraus schloss Rampton, dass die Verwendung polyethnisch geprägter Stile nicht nur ein flüchtiges Merkmal jugendlicher Sprachrepertoires ist. Diese Erkenntnisse stellen Rampton vor ein Konzeptualisierungsproblem, da solche gesprochensprachlichen Phänomene weder eindeutig zur Jugendsprache noch zur multiethnisch geprägten Heteroglossie überwiegend jugendlicher Sprechergruppen gezählt werden können.

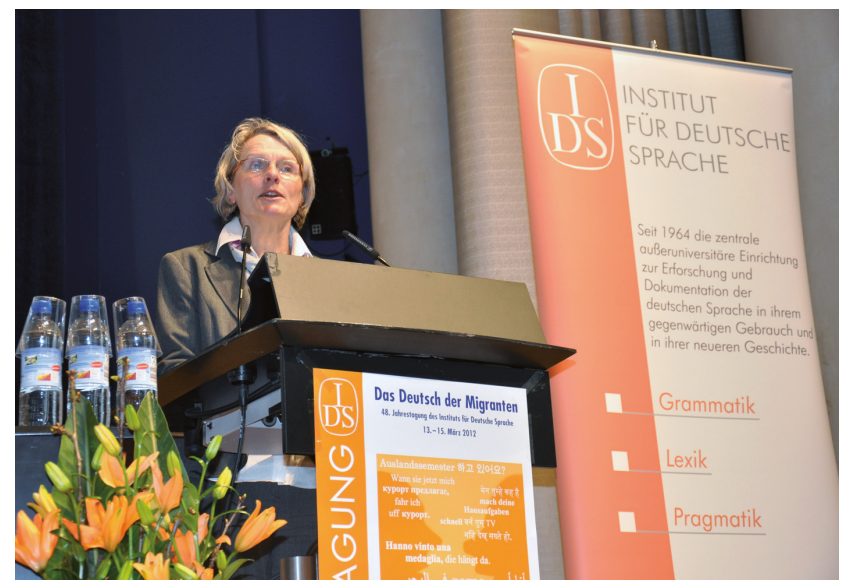

Begrüßung der Tagungsteilnehmer durch Dr. Ulrike Freundlieb, Bürgermeisterin der Stadt Mannheim

Heike Wiese (Potsdam) thematisierte unter dem Titel „Das Potential multiethnischer Sprechergemeinschaften" die Entwicklung eines neuen urbanen Dialekts des Deutschen. Wiese präsentierte dazu einen weiten Dialektbegriff, der nach ihrem Verständnis auch Regiolekte und Soziolekte umfasst. Sie stützte ihre Argumentation auf die Annahme, dass es auf der Ebene empirischer Daten lediglich eine Perspektivenfrage 
sei, inwieweit man multiethnische Sprechweisen als Varietät oder Stil betrachte. Sie schlussfolgerte daraus, dass mit der Bezeichnung, Dialekt' beide Perspektiven zusammengebracht seien. Wiese erläuterte, wie sich dieser neue urbane Dialekt (,Kiezdeutsch') im urbanen Raum entwickle und was diesen Dialekt charakterisiere.

$\mathrm{Zu}$ „Praktiken des Code-switching und Code-mixing in drei türkischen Milieus der 2. Generation - strukturelle und funktionale Aspekte“ referierten Inken Keim und Ibrahim Cindark (Mannheim). Sie präsentierten Ergebnisse der am IDS durchgeführten ethnographisch-soziolinguistischen Untersuchungen zu drei Gruppen von DeutschtürkInnen im Jugend- und Erwachsenenalter, den „türkischen Powergirls“, „den Unmündigen“" und „den Europatürken“. Keim schlussfolgerte, dass

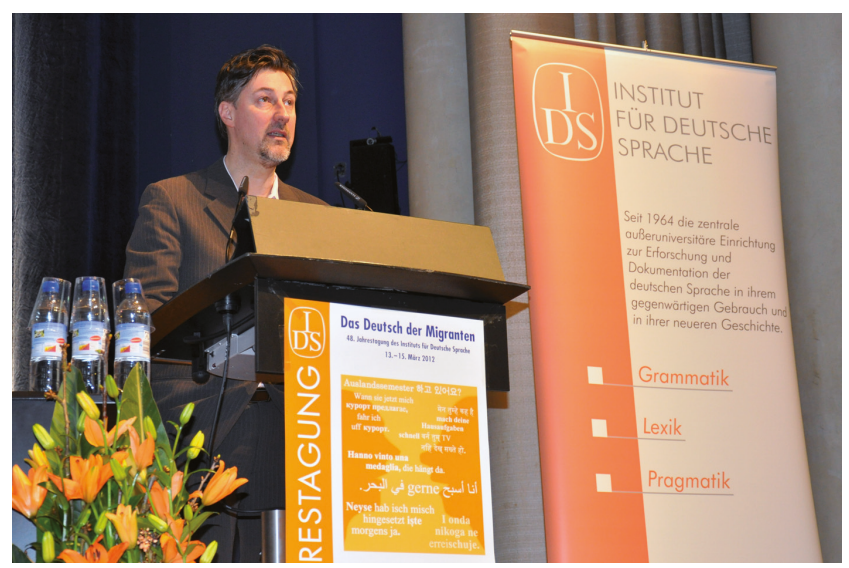

Einführung in das Thema der diesjährigen Tagung durch Prof. Dr. Arnulf Deppermann zipation an Behördenkommunikation befähigt sowie welche sprachlichen Ressourcen und schriftsprachlichen Kompetenzen sie dabei einsetzen. Um feststellen zu können, inwieweit sich autochthone und nicht-autochthone Menschen in dieser Hinsicht unterscheiden, wurden zwei Gruppen anhand sprachlichen Materials aus dem Ruhrgebiet untersucht. Als zentrale Ergebnisse präsentierten Quasthoff und Hoffmann drei Faktoren, die die Wahrnehmung von zufriedenstellender Behördenkommunikation wesentlich beeinflussen: die Autonomie zur selbstständigen Kommunikation mit Behörden, die Transparenz von Behördenabläufen aus Perspektive der Informanten sowie die Durchsetzung der eigenen Interessen.

Auch Ulrich Reitemeier (IDS Mannheim) widmete sich diesem thematischen Schwerpunkt mit einem Vortrag ,Zur kommunikabei den „türkischen Powergirls“ Sprachmischungen zur Abgrenzung gegenüber monolingualen Türken wie Deutschen dienen, als Symbol für die Zugehörigkeit zur 2. Generation gelten und Ausdruck eines neuen Selbstbildes jenseits ethnischer Kategorien sind. Cindark kontrastierte die Befunde der „türkischen Powergirls" mit den Ergebnissen der Untersuchung zu den „Europatürken“ sowie den „Unmündigen“.

Mit „I Germanesi‘. Sprechen mit Migrationshintergrund in drei Generationen" widmete sich Christine Bierbach (Frankfurt am Main) italienischen Migranten in Mannheim. Bierbach präsentierte einen Überblick zu den Sprecherrepertoires der drei untersuchten Generationen und schlussfolgerte, dass die untersuchten Sprecher keinen überwiegend italienisch geprägten Ethnolekt sprechen. Bei der Bezeichnung der Sprechweisen stellte es sich zudem als schwierig dar, einzuschätzen, inwieweit hier von einer Varietät oder einem Stil zu sprechen sei.

Den zweiten thematischen Schwerpunkt des ersten Tagungstages zum Thema „Kommunikation von Migranten mit Behörden“ eröffneten Uta M. Quasthoff und Ludger Hoffmann (Dortmund) mit einem Vortrag zu „Schreiben zwischen Sprachen und Kulturen“ und präsentierten Ergebnisse ihres interdisziplinären Forschungsprojekts „Literacy between Languages and Cultures" (LiLaC). Leitende Fragestellungen waren, was Menschen aus benachteiligten Milieus zur Parti- tiven Realisierung von Differenzorientierung in Situationen zwischen Aussiedlern und Einheimischen". In seinem Vortrag fokussierte Reitemeier Kommunikationssituationen, an denen deutschstämmige Zuwanderer und Einheimische beteiligt waren, und stützte sich auf empirische Daten, die im Zeitraum zwischen 1993 bis 2001 erhoben wurden. In seinen Ausführungen erläuterte Reitemeier die Praktiken des Relevantmachens von Differenz-Identität im Integrationskonflikt zwischen Einheimischen und deutschstämmigen $\mathrm{Zu}$ wanderern. Anhand empirischer Datenbeispiele aus der Aussiedlerberatung und aus einer Leserbriefkontroverse über die Teilnahme an Klassenfahrten erklärte er, wie das Markieren migrationsbedingter Differenzen die Herstellung von Vertrauens- und Kooperationsgrundlagen für weiteres gemeinsames Handeln erschweren kann und wie Praktiken des Relevantmachens von Differenz-Identität die Herstellung von Intersubjektivität beeinträchtigen.

Zum Abschluss des ersten Tagungstages lud Thomas Schmidt (IDS Mannheim) zum Besuch der 3. Fachmesse zur Technologie für mündliche Sprachkorpora ein, die am zweiten Tagungstag ganztägig im Foyer vor dem Musensaal für alle interessierten Teilnehmer offen stand. Bei der diesjährigen Fachmesse für Korpustechnologie waren 19 Aussteller vertreten und präsentierten Neuigkeiten rund um die Erstellung und Auswertung v. a. von Korpora gesprochener Sprache. 
Am Mittwoch wurde der Themenkomplex „Migranten in der Gesellschaft" von Anne Betten (Salzburg) mit einem Vortrag zum Thema „Sprachbiographien deutscher Emigranten: Die ,Jeckes' in Israel zwischen Verlust und Rekonstruktion ihrer kulturellen Identität" eingeleitet. Betten wandte sich primär dem Zusammenhang von Sprachverwendung und Identität bei deutschen Emigranten der 1. und 2. Generation in Israel zu und schilderte, wie biografische Brüche und die Verdrängung der originären Sozialisation zur Übernahme von Perspektiven, Identitätsidealen und -mustern der Aufnahmegesellschaft führen.

Im darauf folgenden Vortrag berichtete Katharina Brizić (Wien) zum Thema „Grenzenlose Biografien und ihr begrenzter Erfolg. Bildungs- und soziale Ungleichheit aus linguistischer Perspektive" über das laufende Projekt „Best Success through Language Loss?" (BEST). Brizić wandte sich der Frage zu, wie Bildungserfolge und mehrsprachige Sprachhintergründe systematisch zusammenhängen. In ihren Ausführungen erläuterte Brizić, wie sie vielsprachige Communities als eine Institution versteht, die soziale Ungleichheit und Machtverhältnisse reproduzieren. Sprachhintergründe von Kindern mit transnational geprägtem Migrationshintergrund veranschaulichte Brizić anhand sprachbiografischer Profile von vier zehnjährigen Schülern. Sie zeigte, wie Kinder mit bis zu sieben Sprachen gleichzeitig konfrontiert sein können und wie sprachpolitische Mechanismen die Sprachwahl innerhalb einer Community massiv beeinflussen.

Dem Thema „SprachGeschichten mit Migrationshintergrund: demografische und biografische Perspektiven auf Sprachkenntnisse und Spracherleben" widmete sich Patrick Stevenson (Southampton) in seinem Vortrag. Stevenson diskutierte, wie die biografische Untersuchung des Spracherlebens einzelner Menschen zu einer Soziolinguistik der Superdiversität beitragen kann und veranschaulichte darauf aufbauend, was über das Ausmaß der sprachlichen Superdiversität in Deutschland in quantitativer Hinsicht bisher bekannt ist. Inspiriert durch eine dokumentarische Erzählung über ein Berliner Mietshaus präsentierte Stevenson erste Tendenzen seiner Studie „Mareschstr. 47“, in der er versucht, ein durch Superdiversität geprägtes Mietshaus zu beschreiben und die durch Transmigration geprägten Lebensgeschichten der einzelnen Bewohner zu erfassen.

Im anschließenden Vortag referierte Rosemarie Tracy (Mannheim) zum Thema „Wer muss was lernen? Stolpersteine einer Bildungsreise“. Sie hob einerseits die Komplexität des Spracherwerbs hervor und betonte andererseits, dass die Komplexität des kommunikativen Verhaltens von Kindern, die insbesondere Lehrkräfte vor ein zentrales Problem im Umgang mit Kindern stellt, die nicht Deutsch als Muttersprache haben. An Beispielen explizierte Tracy die Inadäquatheit gängiger Sprachtests, deren Ergebnisse zentrale Fähigkeiten nicht korrekt erfassen und gerade bei der Begutachtung mehrsprachiger Kinder zu Fehleinschätzungen führen.

Einen thematischen Höhepunkt fand die Tagung in der Podiumsdiskussion, an der Rita Franceschini (Bozen), Konrad Ehlich (Berlin), Jannis Androutsopoulos (Hamburg) und Matthias Makowski (München) unter der Moderation von Ludwig M. Eichinger (IDS) teilnahmen. Die diesjährige Podiums-

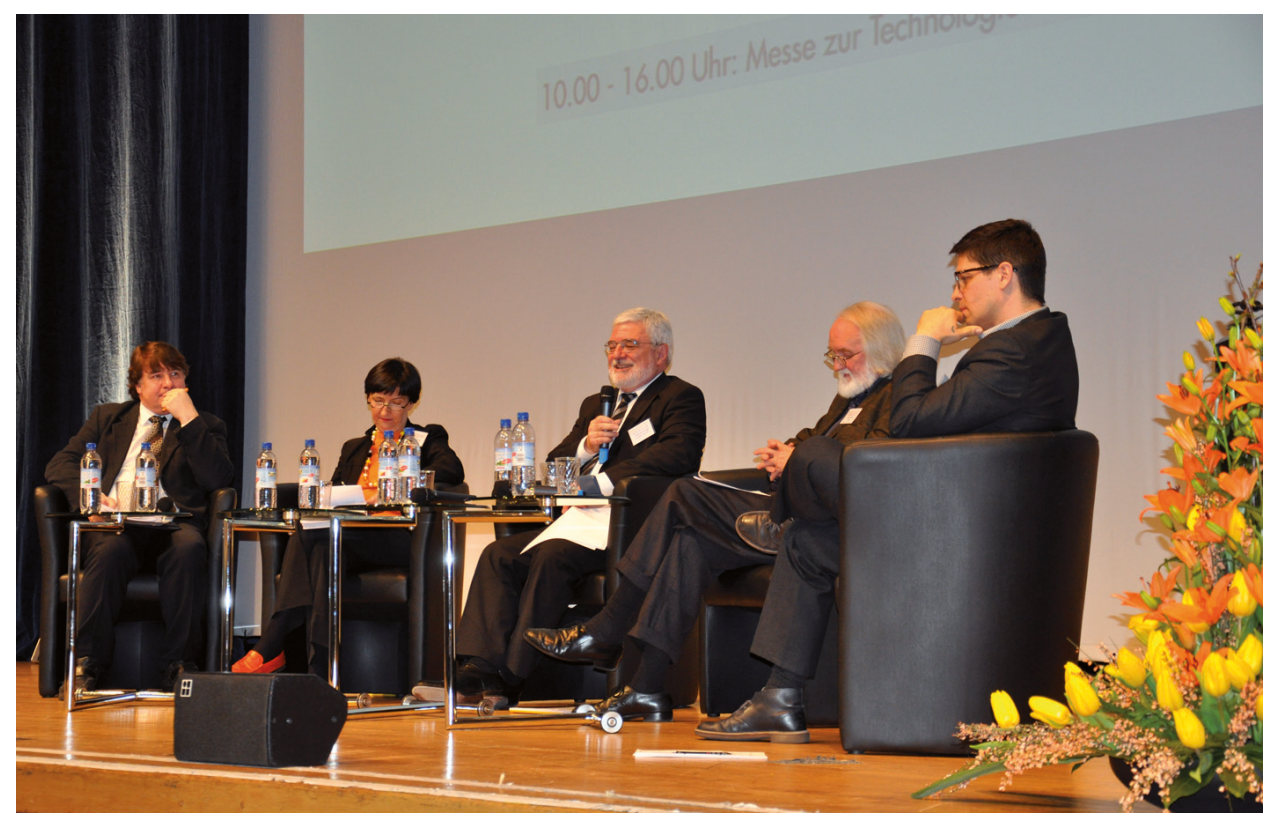

Podiumsdiskussion, von links: Matthias Makowski (München), Rita Franceschini (Bozen), Ludwig M. Eichinger (Direktors des IDS), Konrad Ehlich (Berlin), Jannis Androutsopoulos (Hamburg) diskussion reihte sich nicht in die Tradition vergangener, resümierender Podiumsdiskussionen ein, sondern positionierte sich mit einem kritisch reflektierenden Tenor zum Tagungsthema. Mit einem kurzen und pointierten Statement äußerte sich Androutsopoulos dazu, dass aus Perspektive mehrsprachiger Individuen 
Deutsch immer nur ein Bestandteil komplexer Sprachrepertoires ist und als solches auch immer verwendet wird. Besonders sprach- und medienpolitische Kampagnen wie „Sag's auf Deutsch“ u. a. marginalisieren die komplexen Sprachrepertoires mehrsprachiger Individuen, indem sie Sprachförderung nur aus monolingualer Perspektive akzeptieren. Franceschini empfahl die Bezeichnung, des flexiblen Sprechers', der sich in seinem Repertoire einer Bandbreite von Varietäten bedienen und mit diesen Repertoires in unterschiedlichen Kombinationen umgehen kann. Konrad Ehlich diskutierte, dass das mehrsprachige Individuum in einer Situation der konsolidierten Einsprachigkeit lebt und nun ein neues Konzept gefordert ist, in dem das mehrsprachige Individuum einen neuen Stellenwert einnimmt. Im Anschluss griff Matthias Makowski das Konzept der konsolidierten Einsprachigkeit auf und betonte dessen normgebende Struktur für die Spracharbeit des Goethe-Instituts (GI). Aus Perspektive der Auslandsinstitute des GI stellt das Deutsch der Migranten eine prüf- und erfassbare Kompetenz dar.

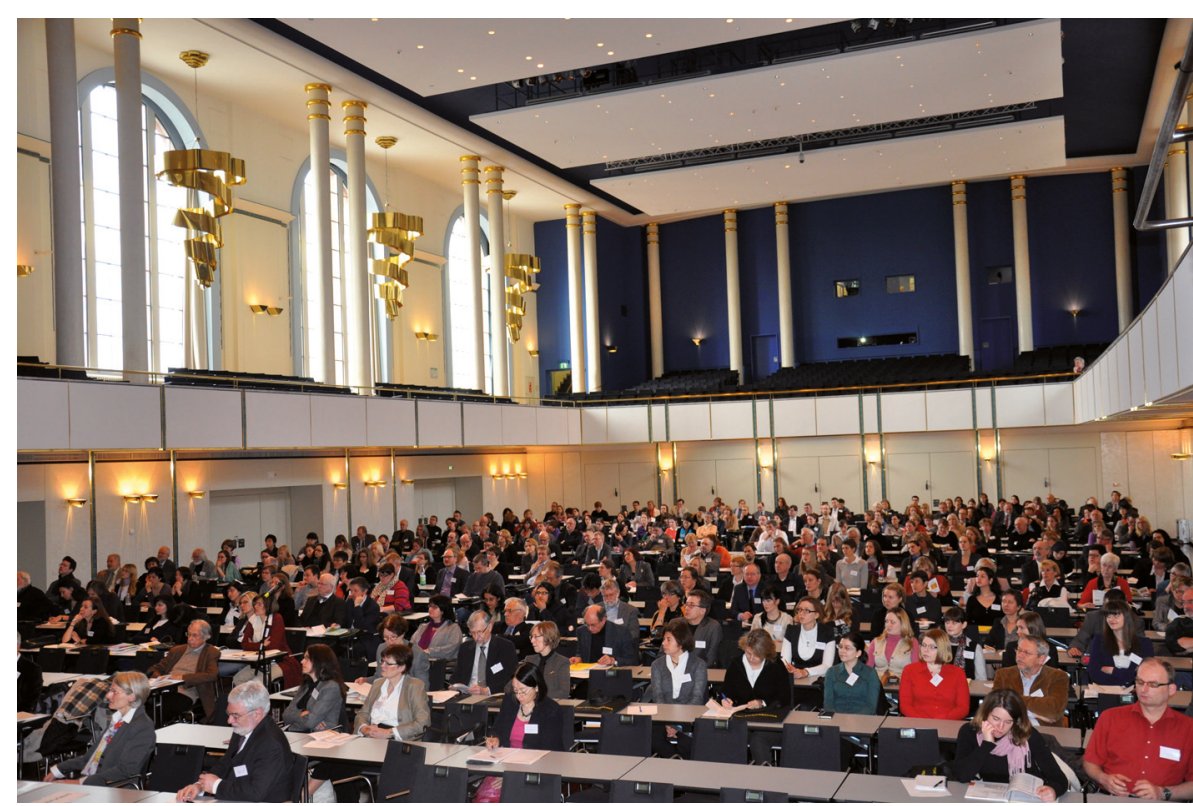

Blick in den Musensaal im Rosengarten Mannheim

Im Anschluss zeichnete die Stadt Mannheim zusammen mit dem Verlag Bibliographisches Institut (Dudenverlag) mit dem Konrad-Duden-Preis 2011 einen bedeutenden Sprachwissenschaftler, Peter Schlobinski, für seine Leistungen zur Erforschung der Internetsprache aus. In der feierlichen Atmosphäre des Rittersaals des Mannheimer Schlosses verlieh Peter Kurz, Oberbürgermeister der Stadt Mannheim, die mit $12.500 €$ dotierte Auszeichnung dem 29. KonradDuden-Preisträger. Mit lobenden Worten begrüßte auch Marion Winkenbach, Geschäftsführerin des Bibliographischen Instituts, den diesjährigen Preisträger. In seiner Laudatio honorierte Ludwig M. Eichinger, Direktor des IDS, stellvertretend für den Preisträger des 28. Konrad-Duden-Preises, das Institut für Deutsche Sprache, Peter Schlobinski als „nicht nur einen der herausragenden unter den jüngeren, sondern einen jüngeren unter den herausragenden Sprachwissenschaftlern".

Der thematische Schwerpunkt des letzten Tagungstages lag auf dem „Erwerb des Deutschen als Zweitsprache", dem sich auch Petra Stanat (Berlin) mit ihrem Vortrag über „Sprachliche Kompetenzen von Heranwachsenden mit Migrationshintergrund und schulischer Erfolg: Befunde der Empirischen Bildungsforschung" widmete. In ihren Ausführungen präsentierte sie Ergebnisse des Projekts „Bedeutung und Form“ (BeFo), durch welches Kindern mit Migrationshintergrund zu besseren Deutschkompetenzen verholfen werden soll. Stanats erste Ergebnisse zeigten allerdings keine signifikanten Effekte der Förderung auf die sprachliche Kompetenzsteigerung.

Im darauffolgenden Vortrag zu „Silben und Füße im Deutschen und Türkischen (Verdeckte) Probleme beim Orthographieerwerb des Deutschen durch türkischsprachige Lerner/ innen" verwies Ursula Bredel (Hildesheim) auf die Tatsache, dass der Erwerb der geschriebenen Sprache vor allem bei L2Sprechern ab Schuleintritt mit dem der gesprochenen Sprache ein logisches und zeitliches Miteinander bildet. Sie diskutierte anhand empirischer Daten, wie sich der Schriftspracherwerb bei Schulkindern im Detail vollzieht, welche Bedeutung Rhythmus und Akzent beim Orthografieerwerb zukommt und wie im Schriftspracherwerb als Zweitspracherwerb orthografiekodierte Strukturen erworben werden.

Ulrich Mehlem (Frankfurt am Main) erläuterte in seinem Vortrag sehr anschaulich, wie Grundschüler türkischer Herkunftssprache über einen Stummfilm auf Deutsch schreiben und erzählen. In seinen Ausführungen präsentierte er Ergebnisse des Projekts „Literacy Acquisition in Schools in the Context of Migration and Multilingualism“ (LAS). Der Schwerpunkt lag dabei auf dem Versuch, Mündlichkeit und Schriftlichkeit auf konzeptueller Ebene zu vergleichen und diese Ergebnisse hinsichtlich gleicher sprachlicher Einheiten nach bestimmten Kategorien auszuwerten. In seinem Fazit resümierte Mehlem, dass die Erzählung als mündli- 
che Ressource Kinder stärker zum schriftsprachlichen Diskurs führen kann.

Unter dem Titel „,Meine Muttersprache ist Russisch, aber ausdrücken kann ich mich besser auf Deutsch'. Die Rolle der Herkunfts- und der Umgebungssprache bei Jugendlichen aus russischsprachigen Familien“" berichtete Tanja Anstatt (Bochum) in ihrem Vortrag von russischsprachigen Jugendlichen, die das Fach „Russisch als zweite Fremdsprache“ an einer Schule in Nordrhein-Westfalen besuchen. Anstatt präsentierte erste Ergebnisse und verwies darauf, dass ihre Daten nicht repräsentativ seien. Sie schlussfolgerte, dass der Erwerb des Deutschen für die untersuchten Jugendlichen unproblematisch verlaufen sei, während das Russische auch weiterhin die Hauptkommunikationssprache mit den Eltern bleibt.

Im letzten Vortrag der diesjährigen Jahrestagung referierte Petra Schulz (Frankfurt am Main) zu „Wer versteht wann was? Sprachverstehen im frühen Zweitspracherwerb des Deutschen“. Darin beleuchtete sie die semantischen und syntaktischen Aspekte von WFragen, die für die Erforschung des Sprachverstehens früher Zweitsprachlerner relevant sind. Im Anschluss präsentierte Schulz ihre Ergebnisse eines Experiments zu exhaustiven W-Fragen, einem Baustein ihres Gesamtprojekts „The Role of Migration Background and Language Impairment in Language Achievement" (MILA). Das Ergebnis ihres Sprachexperiments zeigt, dass Kinder mit frühem Zweitspracherwerb eine ähnliche Entwicklung im Erwerb von Satzstrukturen wie Kinder mit einem ungestörten Erstspracherwerb aufweisen.

Das Schlusswort zur 48. IDS-Jahrestagung gebührte dem Direktor des Instituts für Deutsche Sprache. Herzlich bedankte sich Ludwig M. Eichinger bei allen Referenten für ihre Beiträge und verabschiedete alle Teilnehmer mit einer Ankündigung zu „Sprachverfall? Sprachliche Dynamik, Sprachwandel, sprachliche Variation", einem brisanten und nicht minder relevanten Thema, das im kommenden Jahr den Rahmen für die 49. Jahrestagung bilden wird, die vom 12.-14. März 2013 im Mannheimer Rosengarten stattfinden wird.

Die Autorin ist wissenschaftliche Mitarbeiterin am Institut für Deutsche Sprache in Mannheim.

Fotos: Annette Trabold 